\title{
Towards signal processing assisted hardware for continuous in-band electrode impedance monitoring
}

DOI:

10.1109/ISCAS.2017.8050348

\section{Document Version}

Accepted author manuscript

Link to publication record in Manchester Research Explorer

\section{Citation for published version (APA):}

Kohli, S., \& Casson, A. (2017). Towards signal processing assisted hardware for continuous in-band electrode impedance monitoring. In Circuits and Systems (ISCAS), 2017 IEEE International Symposium on https://doi.org/10.1109/ISCAS.2017.8050348

\section{Published in:}

Circuits and Systems (ISCAS), 2017 IEEE International Symposium on

\section{Citing this paper}

Please note that where the full-text provided on Manchester Research Explorer is the Author Accepted Manuscript or Proof version this may differ from the final Published version. If citing, it is advised that you check and use the publisher's definitive version.

\section{General rights}

Copyright and moral rights for the publications made accessible in the Research Explorer are retained by the authors and/or other copyright owners and it is a condition of accessing publications that users recognise and abide by the legal requirements associated with these rights.

\section{Takedown policy}

If you believe that this document breaches copyright please refer to the University of Manchester's Takedown Procedures [http://man.ac.uk/04Y6Bo] or contact uml.scholarlycommunications@manchester.ac.uk providing relevant details, so we can investigate your claim.

\section{OPEN ACCESS}




\title{
Towards signal processing assisted hardware for continuous in-band electrode impedance monitoring
}

\author{
(Invited Paper)
}

\author{
Siddharth Kohli and Alexander J. Casson \\ School of Electrical and Electronic Engineering, The University of Manchester, Manchester, UK \\ Email: siddharth.kohli@postgrad.manchester.ac.uk, alex.casson@manchester.ac.uk
}

\begin{abstract}
Maintaining low and stable electrode contact impedances is critical for obtaining high quality signals in out-ofthe-lab EEG units. Current EEG units measure the impedance of the electrode contacts by injecting an out-of-band, typically $1 \mathrm{kHz}$, current into the head. This high frequency component is easily isolated from the true EEG to avoid introducing artifacts, but does not give direct information on the contact impedance at the wanted cortical frequencies, typically at 5-30 Hz. This paper investigates two techniques for allowing simultaneous impedance measurements at 5-30 $\mathrm{Hz}$ for the first time. One method uses digital processing for removing the EEG artifact that continuous in-band impedance monitoring produces. The other uses a new $36 \mathrm{nW}$ notch filter for removing the interference. Both are shown to allow impedance monitoring at $5-30 \mathrm{~Hz}$ while leaving minimal residual artifacts in the collected EEG traces.
\end{abstract}

\section{INTRODUCTION}

The electroencephalogram (EEG) is a widely used tool for neuroimaging. It is based upon placing small metal electrodes on the scalp and recording the very small electrical potentials outside of the body due to neuronal action within the brain. It is in principal a small, portable technology and ambulatory EEG units which do not require long recording wires have been available for many years [1]. In the last decade there has been significant interest in wearable EEG devices that are even more miniaturised, easy to use, and allow high quality, real world, neuroimaging for the first time [2]. However, despite this interest and significant progress, there remain many challenges before true real world EEG is realised [3]. For improving data quality and resistance to artifacts the performance of the electrode contact with the scalp is critical. In the ideal case the impedances of all electrodes are equal and this minimises the common mode-to-differential conversion of external interference sources such as 50/60 Hz pick-up and motion. However, historically it has not been possible to measure the impedance of an EEG electrode simultaneously with signal collection. Impedance measurements, and correcting mismatches by adjusting the physical connections, have only been done at the start/end of a recording.

Recently, simultaneous EEG monitoring and impedance measurement has been proposed by recording EEG in the usual manner, and simultaneously injecting a small current at $\sim 1 \mathrm{kHz}$, out of the EEG frequency range of interest [4], Fig. 1. The out-of-band signal can be isolated in the instrumentation electronics and used to calculate the contact impedance at $1 \mathrm{kHz}$ while leaving the lower frequency EEG trace unaffected. However, this relies on having a good model of the electrode-scalp contact to allow the impedance at physiological frequencies $(5-30 \mathrm{~Hz})$ to be inferred. This is challenging, because the actual electrode contact model varies over time with the amount of hair present, the scalp condition, the amount of sweat and similar. In this paper we investigate techniques to allow the contact impedance at $5-30 \mathrm{~Hz}$ to be measured directly while leaving minimal residual artifacts in the collected EEG trace. In Section II we present a new notch filter for removing in-band interference, and a signal processing enhanced method using a combination of hardware and software to remove the artifact. The performance is assessed in Section III and the second method in particular allows a continuous EEG impedance measurement, giving greater realtime insights into the quality of the EEG, and is the first step towards a dynamic EEG front-end which could alter its electrode impedance in real-time to automatically balance the impedance between electrodes.

\section{ARTIFACT REMOVAL METHODS}

The basis for simultaneous EEG and contact impedance measurement is shown in Fig. 1. In conjunction with a standard EEG amplifier a current source is connected to the electrode and drives a small current into the body. A typical waveform used for the impedance signal at $15 \mathrm{~Hz}$ is shown in Fig. 2a. This signal mixes with the true EEG and when both are recorded at the same time leads to an artifact corrupted EEG trace, Fig. 2b. As the frequency of the impedance signal is known it can be isolated and the recorded amplitude and phase at this frequency used to calculate the impedance. Conventionally the EEG collected at the same time is not used as it contains artifacts from the impedance measurement. Such artifacts can be avoided by performing the impedance measurements at out-of-band frequencies, typically $1 \mathrm{kHz}$. Here we consider two methods for removing impedance measurement artifacts applied in-band at frequencies from 5 to $30 \mathrm{~Hz}$.

\section{A. Notch filter method}

The first approach is based upon a new ultra low power high order notch filter for removing the specific in-band frequency that the stimulation artifact is present at. For low power, low voltage, low frequency operation a $g_{m} \mathrm{C}$ filter structure is used as its power consumption is directly proportional to the cut-off frequency, which is itself very low $(5-30 \mathrm{~Hz}$ for in-band EEG). A fully differential fourth order topology is shown in Fig. 3 with $Q=0.735$. The design is based on a doubly terminated LC ladder prototype as this structure gives the minimum sensitivity to process and device mismatch when operating in weak inversion [5]. 


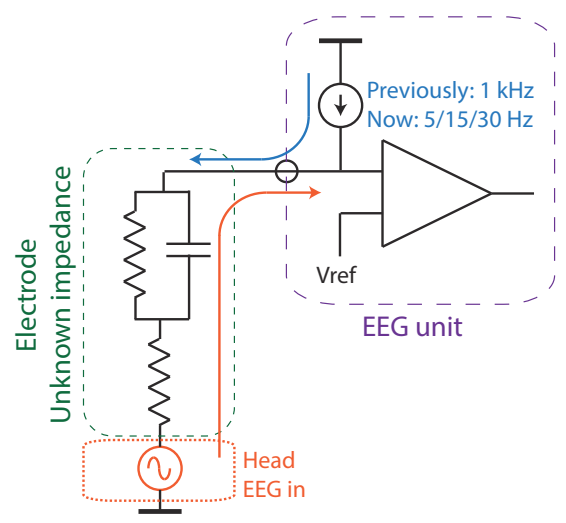

Fig. 1. Simultaneous EEG recording and contact impedance measurement drives the body with a small known current, with the waveform in Fig. 2a.
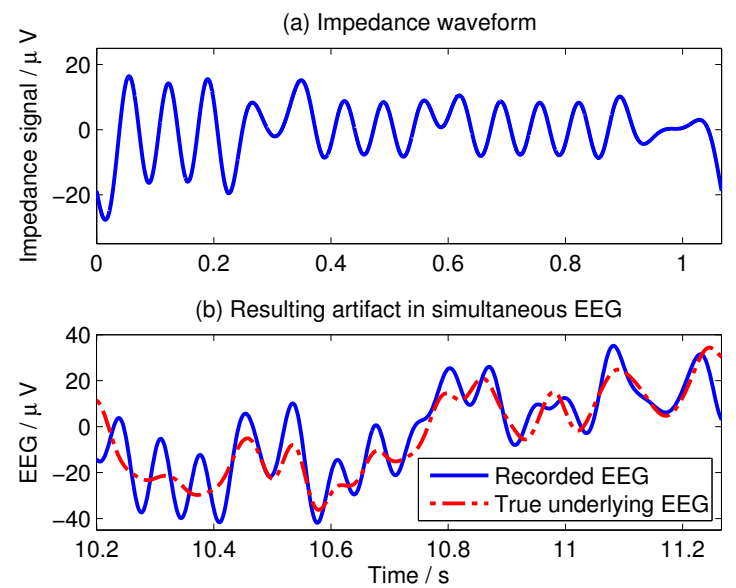

Fig. 2. (a) $15 \mathrm{~Hz}$ signal used for measuring contact impedance. In practice the superposition of two components either side of $15 \mathrm{~Hz}$ are used to give improved robustness. (b) Simultaneously collected EEG (recorded using the head model in Section III) shows artifacts due to the impedance measurement.

Capacitors are sized for a transconductance in the range 1-10 nS, and the used transconductor is shown in Fig. 4. For low power, low transconductance operation it has a folded cascode structure with input cross-coupling for transconductance reduction [6]. A nominal bias current of $512 \mathrm{pA}$ is used, which can be tuned to provide the wanted impedance rejection frequency. This means that transistors are biased in the deep weak inversion region [7], minimising power at the cost of bandwidth. The low frequency nature of EEG $(<100 \mathrm{~Hz})$ means that the required bandwidth is readily achieved via suitable transistor sizing. The whole circuit operates from a $1.3 \mathrm{~V}$ supply so can be directly driven from a single coin cell battery. Implemented in a triple well, $0.18 \mu \mathrm{m}$ CMOS process with MIM capacitors the layout is shown in Fig. 5 and extracted simulation performance in Table I.

\section{B. Superposition of Moving Averages method}

The second approach is based upon digital processing. Superposition of Moving Averages (SMA) was originally developed for removing artifacts of transcranial a.c. current stimulation (tACS) from simultaneous EEG measurements [8]. In tACS rubber scalp electrodes non-invasively inject currents into the head at cortical frequencies, typically between 5 and
TABLE I. PERFORMANCE OF THE $4^{\mathrm{TH}}$ ORDER NOTCH FILTER.

\begin{tabular}{cc}
\hline CMOS process & $0.18 \mu \mathrm{m}$ \\
Supply voltage & $1.3 \mathrm{~V}$ \\
Typical bias current & $512 \mathrm{pA}$ \\
Area & $0.42 \mathrm{~mm}^{2}$ \\
Power consumption & $35.8 \mathrm{nW}$ \\
Signal input range & $100 \mathrm{mV}_{\mathrm{pp}}$ \\
THD (100 mVpp, 10 Hz in) & $-52.5 \mathrm{~dB}$ \\
Dynamic range & $52 \mathrm{~dB}$ \\
Tuning range & $2-720 \mathrm{~Hz}$ \\
\hline
\end{tabular}

$40 \mathrm{~Hz}$, in order to directly modulate the on-going neural activity. Typically currents up to $2 \mathrm{~mA}_{\mathrm{pp}}$ are used, and these produce a very large artifact signal which obscures the EEG trace. In principle in-band impedance measurements are exactly the same, but inject much smaller sub- $\mu$ A currents which do not modulate the ongoing operation of the brain, and give a similar but smaller artifact to reject.

The SMA formulation used here is given in Fig. 6 [8]. The length of the impedance artifact is known and so the EEG trace is segmented in to $N$ epochs of this duration, and $M$ such epochs are averaged together such that the EEG signal content averages to zero while the amplitude of the periodic impedance artifact is maintained. This allows the generation of a channel and time specific template of the impedance artifact, which evolves with time and intrinsically scales with the number of EEG channels used. The scheme is formulated so that the artifact removed EEG is generated by subtracting the current artifact template from the current EEG. In this work this is done in software in the digital domain. It is anticipated that in the future this can be implemented as in-the-loop signal processing, suppressing the artifact as it is collected, Fig. 7. Here $M$ is selected to be $5 \%$ of $N$ (the total number of impedance measurements) as a suitable trade-off between the number of averages taken and the time localization of the artifact template.

\section{PERFORMANCE COMPARISON}

To assess performance an EEG head model phantom, similar to that in [9], was used to simulate simultaneous EEG and in-band impedance measurements. Electrodes were implanted inside head shaped conductive gelatine and prerecorded EEG sized signals played into the model from a data acquisition unit. This allowed known EEG signals to be present at the surface of the head and provide a known comparison signal for assessing the EEG data quality during impedance measurement. The experiment set up is shown in Fig. 8 where EEG electrodes are held in place using EC2 adhesive (Natus Medical, USA). Two electrodes on the midline $(\mathrm{FCz}, \mathrm{Cz})$ were used as EEG ground and reference and connected to an Enobio (Starlabs, Spain) ambulatory EEG unit, with a recording channel on the rear of the head in location P4. These were simultaneously connected to a SIGGI (EasyCap, Germany) stand alone impedance meter which injected an impedance current at the desired frequency and allowed the impedances to be measured during the EEG acquisition. The collected signals were then processed using the notch filter approach or the SMA approach to remove the artifact of the impedance measurement. 


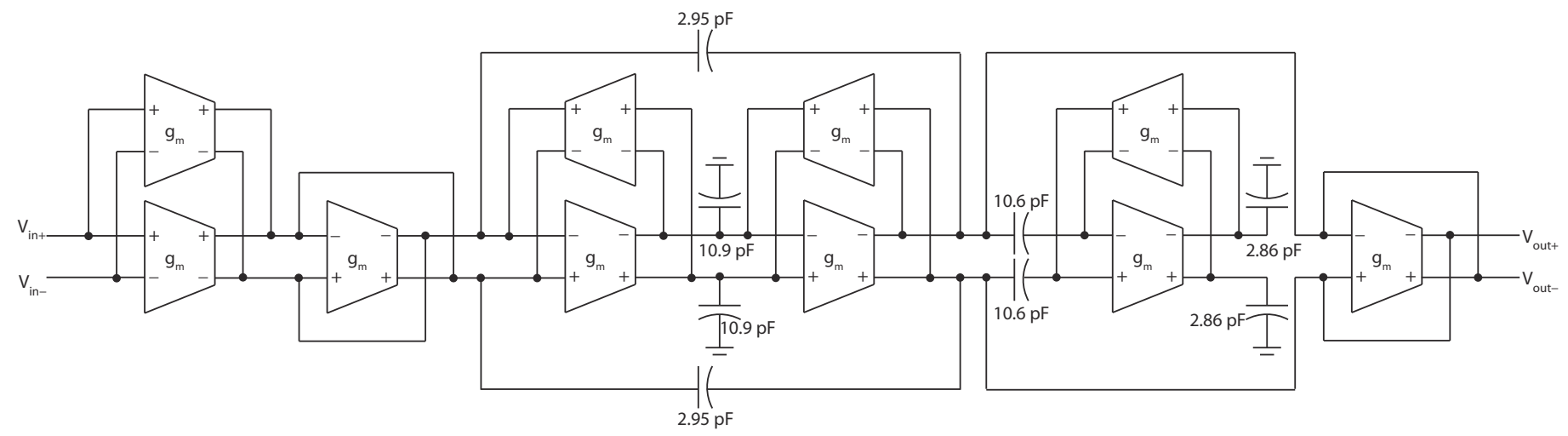

Fig. 3. Fourth order $g_{m} \mathrm{C}$ notch filter circuit based on a doubly terminated LC ladder. All transconductors are the same (Fig. 4).

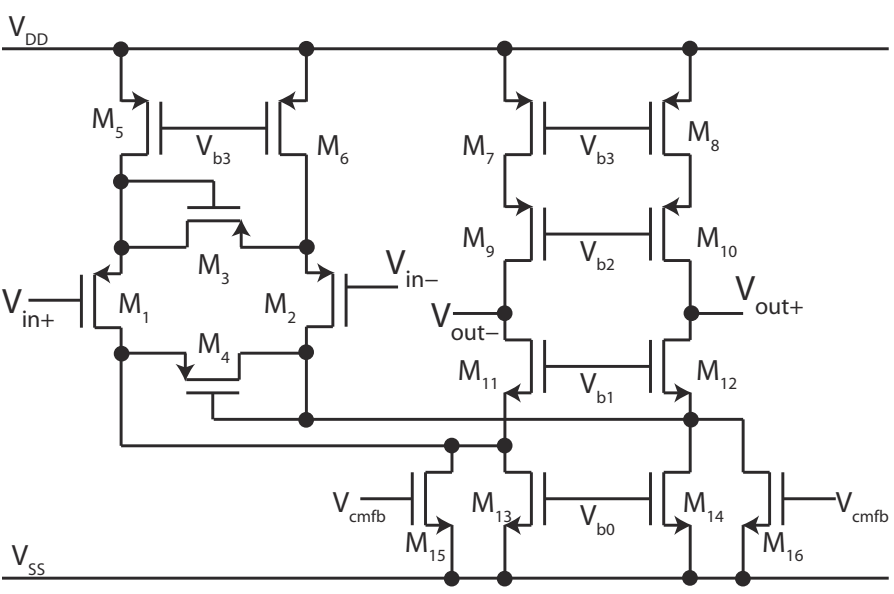

Fig. 4. 1-10 nS transconductor with folded cascode, input cross-coupling working in deep weak inversion for low power, low transconductance operation.

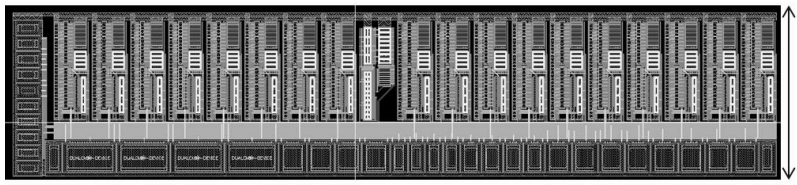

Fig. 5. Layout of the proposed notch filter.

In all test set ups the impedance was measured continuously, with impedance values being constant at approximately $1.2 \mathrm{k} \Omega$. An illustrative example of the impedance artifact removal process is shown in Fig. 9 for an in-band impedance measurement at $5 \mathrm{~Hz}$ which highly overlaps with cortical frequencies of interest in the $5-30 \mathrm{~Hz}$ range. Fig. 9a shows a section of EEG data recorded from the head model, which includes artifacts from the impedance measurement process (as in Fig. 2). Fig. 9b shows the known EEG trace which would be recorded in the artifact free case, and Fig. 9c and d show the processed EEG data after SMA and notch filter methods respectively. Both Fig. 9c and d follow the general trend of the EEG signal shown in Fig. 9b, for example with a large negative component at time $47 \mathrm{~s}$. Within this, the SMA method closely follows the desired EEG trace, with more residual artifacts present after notch filtering, which is expected as it also removes cortical frequencies of interest.

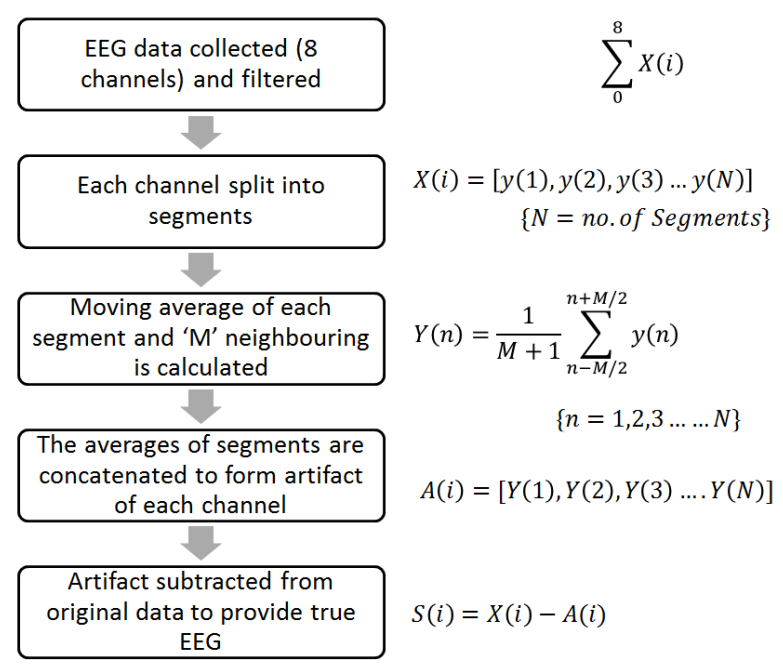

Fig. 6. Algorithm for SMA. Data from each channel is separately segmented before a moving average artifact template is calculated, which is then subtracted from the original data recorded for that channel.

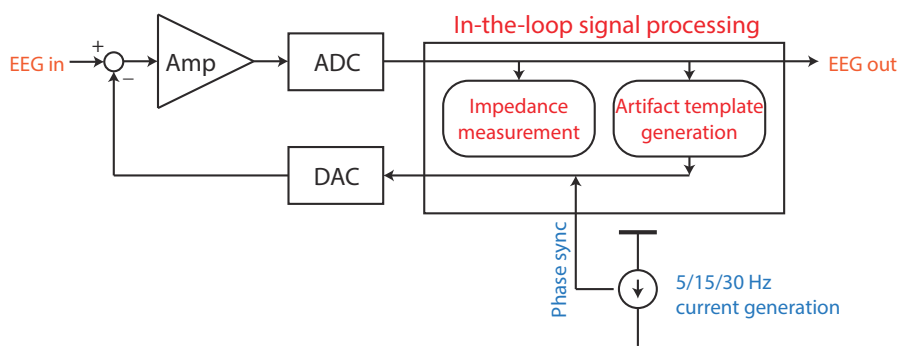

Fig. 7. Proposed signal processing assisted amplifier for in-band impedance measurements.

The performance is quantified in Table II for in-band impedance measurements at 5,15 and $30 \mathrm{~Hz}$. Table II measures the correlation coefficient between the known EEG trace and the artifact processed EEG traces over 2 minute recordings which include pre-recorded alpha activity, that is, bursts of EEG in the $8-12 \mathrm{~Hz}$ range. This input is deliberately chosen to be directly adjacent to the impedance measurement frequencies giving a challenging signal recording and artifact rejection case. Using SMA, for $5 \mathrm{~Hz}$ and $15 \mathrm{~Hz}$ impedance measurements the correlations are very high, $>0.9$, showing that the 


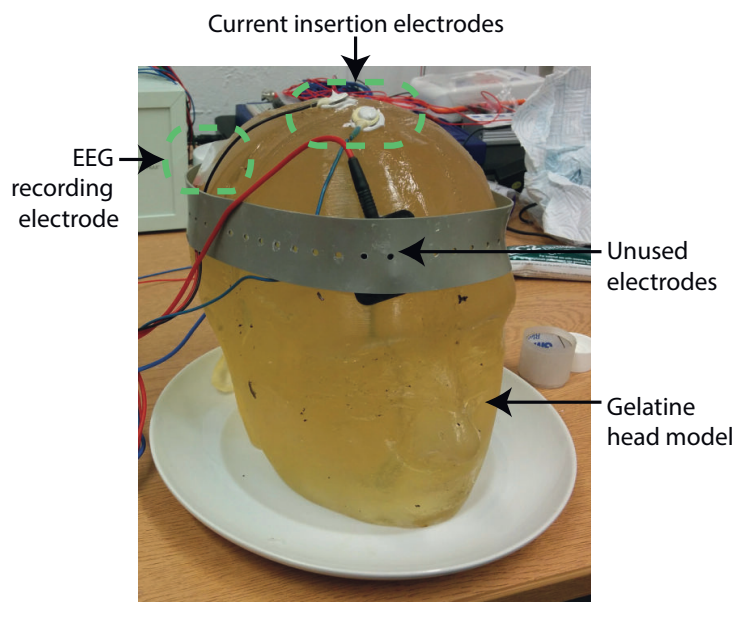

Fig. 8. Head phantom used to provide a known reference EEG during simultaneous EEG and in-band impedance measurements.

(a) Recorded EEG with impedance artifacts

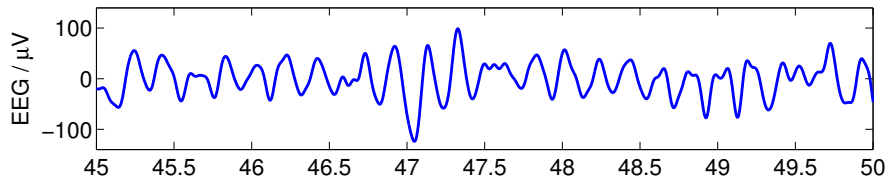

(b) Known EEG signal

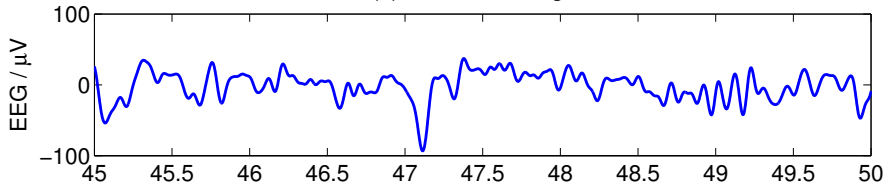

(c) Artifact removed EEG via SMA

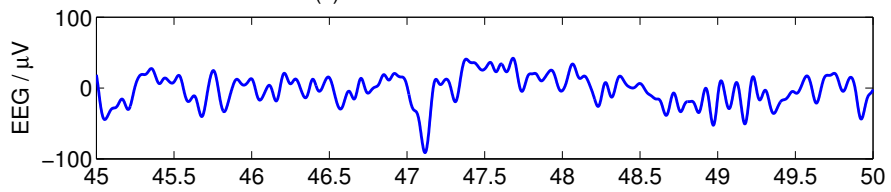

(d) Artifact removed EEG via notch filter

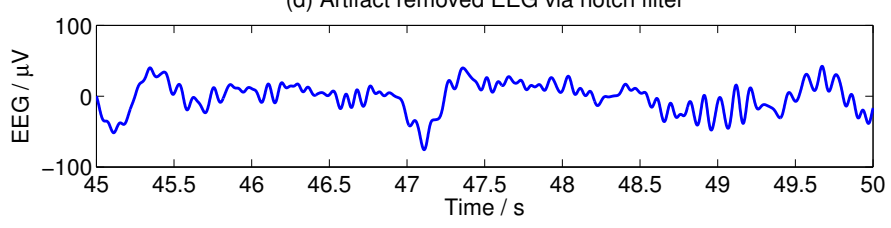

Fig. 9. Example removal of impedance artifact for a $5 \mathrm{~Hz}$ in-band impedance measure.

EEG is correctly recorded despite the presence of an in-band impedance artifact in the raw trace. The performance of the notch filter is worse, particularly for the low $5 \mathrm{~Hz}$ impedance measurement. To put these figures in context, typical correlation coefficients when comparing the performance of different EEG electrode types are: $>0.93$ [10]; 0.89 [11]; 0.83 [12]; $0.81-0.98$ [13]; 0.68-0.90 [14]; 0.39-0.85 [15]. Using both in-band impedance methods the correlation coefficients are larger than these, indicating a minimal loss of information, equivalent to changing the electrode type used. The SMA approach consistently outperforms the notch filtering method with high correlation coefficients obtained at all impedance measurement frequencies.
TABLE II. CORRELATION COEFFICIENTS BETWEEN THE KNOWN EEG TRACE AND THE ARTIFACT PROCESSED TRACES.

\begin{tabular}{cccc}
\hline \multirow{2}{*}{ Method } & \multicolumn{3}{c}{ Impedance measurement frequency / Hz } \\
& 5 & 15 & 30 \\
\hline SMA & 0.94 & 0.94 & 0.88 \\
Notch & 0.79 & 0.92 & 0.82 \\
\hline
\end{tabular}

\section{CONCLUSION}

Electrode contact impedance measurements are an important assessment of data quality in EEG recordings, but have not previously been possible to obtain simultaneously with EEG at the wanted frequencies in the $5-30 \mathrm{~Hz}$ range. This paper has demonstrated in-band simultaneous impedance monitoring for the first time and showed that minimal residual artifacts are introduced in the EEG trace, with correlations compared to a gold standard reference of up to 0.94 .

\section{REFERENCES}

[1] E. Waterhouse, "New horizons in ambulatory electroencephalography," IEEE Eng. Med. Biol. Mag., vol. 22, no. 3, pp. 74-80, 2003.

[2] A. J. Casson, D. C. Yates, S. J. Smith, J. S. Duncan, and E. RodriguezVillegas, "Wearable electroencephalography," IEEE Eng. Med. Biol. Mag., vol. 29, no. 3, pp. 44-56, 2010.

[3] V. Mihajlovic, B. Grundlehner, R. Vullers, and J. Penders, "Wearable, wireless eeg solutions in daily life applications: What are we missing?" IEEE. J. Biomed. Health Inform., vol. 19, no. 1, p. 621, 2015.

[4] M. Guermandi, R. Cardu, E. F. Scarselli, and R. Guerrieri, "Active electrode IC for EEG and electrical impedance tomography with continuous monitoring of contact impedance," IEEE Trans. Biomed. Circuits Syst., vol. 9, no. 1, p. 2133, 2015.

[5] A. J. Casson and E. Rodriguez-Villegas, "A review and modern approach to LC ladder synthesis," J. Low Power Electron. Appl., vol. 1, no. 1, pp. 20-44, 2011.

[6] D. Stefanovic and M. Kayal, Structured analog CMOS design. Berlin: Springer, 2008.

[7] A. J. Casson and E. Rodriguez-Villegas, "A $60 \mathrm{pW} g_{m} C$ Continuous Wavelet Transform circuit for portable EEG systems," IEEE J. SolidState Circuits, vol. 46, no. 6, pp. 1406-1415, 2011.

[8] S. Kohli and A. J. Casson, "Removal of transcranial a.c. current stimulation artifact from simultaneous EEG recordings by superposition of moving averages," in IEEE EMBC, Milan, August 2015.

[9] A. S. Oliveira, B. R. Schlink, W. D. Hairston, P. Konig, and D. P. Ferris, "Induction and separation of motion artifacts in EEG data using a mobile phantom head device," PLoS One, vol. 11, no. 1, p. e0145875, 2016

[10] J. Xu, R. F. Yazicioglu, B. Grundlehner, P. Harpe, K. A. A. Makinwa, and C. Van Hoof, "A $160 \mu \mathrm{W}$ 8-channel active electrode system for EEG monitoring," IEEE Trans. Biomed. Circuits Syst., vol. 5, no. 6, pp. 555-567, 2011.

[11] R. Matthews, N. J. McDonald, P. Hervieux, P. J. Turner, and M. A. Steindorf, "A wearable physiological sensor suite for unobtrusive monitoring of physiological and cognitive state," in IEEE EMBC, Lyon, August 2007.

[12] G. Gargiulo, P. Bifulco, R. A. Calvo, M. Cesarelli, C. Jin, and A. van Schaik, "A mobile EEG system with dry electrodes," in IEEE BioCAS, Baltimore, November 2008.

[13] IMEC. (2012) Holst centre and panasonic present wireless low-power active-electrode EEG headset. [Online]. Available: http://www.imec.be/

[14] S. Patki, B. Grundlehner, A. Verwegen, S. Mitra, J. Xu, A. Matsumoto, R. F. Yazicioglu, and J. Penders, "Wireless EEG system with real time impedance monitoring and active electrodes," in IEEE BioCAS, Hsinchu, November 2012.

[15] J. R. Estepp, J. C. Christensen, J. W. Monnin, I. M. Davis, and G. F. Wilson, "Validation of a dry electrode system for EEG," in Proc. HFES, San Antonio, October 2009. 